\title{
PRODUCCIÓN ACADÉMICA Y EDUCATIVA DE UN ESTUDIANTE DE MAESTRÍA DEL EPT EN IFAP MACAPÁ, AMAPÁ, AMAZÔNIA, BRASIL
}

\section{ARTÍCULO ORIGINAL}

VIANA, Valderi Nascimento ${ }^{1}$, FECURY, Amanda Alves², OLIVEIRA, Euzébio de ${ }^{3}$, DENDASCK, Carla Viana ${ }^{4}$, DIAS, Claudio Alberto Gellis de Mattos ${ }^{5}$

VIANA, Valderi Nascimento. Et al. Producción académica y educativa de un estudiante de maestría del EPT en IFAP Macapá, Amapá, Amazônia, Brasil. Revista Científica Multidisciplinar Núcleo do Conhecimento. Año 06, Ed. 06, Vol. 08, págs. 186 y 200. Junio de 2021. ISSN: 2448-0959, Enlace de acceso: https://www.nucleodoconhecimento.com.br/educacion-es/estudiante-de-maestria, DOI: 10.32749/nucleodoconhecimento.com.br/educacion-es/estudiante-de-maestria

\section{RESUMEN}

La producción científica dentro de los cursos de pregrado y posgrado tiene su importancia para la construcción de una nueva forma de pensar del estudiante. El Máster Profesional en Formación Profesional en Red Nacional (ProfEPT) une los contenidos disciplinarios, los conocimientos pedagógicos y la producción académica final y los vincula a situaciones reales de la práctica educativa, y el trabajo final puede

\footnotetext{
${ }^{1}$ Educador Físico, Especialista en Fisiología del Ejercicio y Nutrición Deportiva (FAMA), Estudiante del Programa de Postgrado en Educación Profesional y Tecnológica (PROFEPT IFAP).

${ }^{2}$ Biomédico, Doctor en Enfermedades Tropicales, Profesor e investigador del Curso Médico del Campus Macapá, Universidad Federal de Amapá (UNIFAP).

${ }^{3}$ Biólogo, Doctor en Enfermedades Tropicales, Profesor e investigador del Curso de Educación Física de la Universidad Federal de Pará (UFPA).

${ }^{4}$ Teólogo, Doctor en Psicoanálisis Clínica. Ha estado trabajando durante 15 años con Metodología Científica (Método de Investigación) en la Guía de Producción Científica de estudiantes de Maestría y Doctorado. Especialista en Investigación de Mercado e Investigación centrado en la salud. Estudiante de doctorado en Comunicación y Semiótica (PUC SP).

${ }^{5}$ Biólogo, Doctor en Teoría e Investigación del Comportamiento, Profesor e investigador del Curso de Grado de Química del Instituto de Educación Básica, Técnica y Tecnológica de Amapá (IFAP) y del Programa de Posgrado en Educación Profesional y Tecnológica (PROFEPT IFAP).

RC: 89147

Enlace de acceso: https://www.nucleodoconhecimento.com.br/educacion-es/estudiante-de$\underline{\text { maestria }}$
} 
ser presentado de diversas maneras (artículos, libros, productos educativos). La producción académica, tanto del asesor como del orientador, además de la importancia relacionada con la divulgación científica, tiene peso en la evaluación del programa y también en su mantenimiento. El objetivo de este trabajo es mostrar cuantitativamente la producción académica y educativa de un estudiante de maestría en Educación Profesional y Tecnológica (EPT) del Instituto de Educación, Ciencia y Tecnología (IFAP) de Macapá, Amapá, Amazônia, Brasil. Durante el máster, la producción científica en diversas formas resultó necesaria durante el proceso de formación. Uniendo la teoría con la práctica de la escritura, facilitó la producción de la disertación que debía presentarse al final del curso. A través del proceso de organización, la planificación de las ideas dio lugar a un nuevo pensamiento estructurado de acuerdo con los estándares de la academia, pero que no se limita a este espacio y las normas. La producción durante la maestría busca contribuir cuantitativa y cualitativamente a la producción científica de la región norte, impactando positivamente en ella y en el programa de maestría. Además de la importancia en la contribución de la creación y divulgación científica, las producciones tienden a impactar positivamente en la evaluación y mantenimiento del programa PROFEPTFIPA, realizado por la CAPES.

Palabras clave: EPT, Difusión, Producción Científica, PRORFEPT.

\section{INTRODUCCÍON}

Uniendo instrumentos teóricos y metodológicos oportunidades la realización de nuevos proyectos, el estudiante deja de ser sólo un repetidor y comienza a producir conocimiento, contribuyendo con un tema o área específica. A través de la construcción del propio pensamiento, producen nuevas actitudes y comportamientos, es decir, expone una idea plausible y estructurada, presentando juicios de valor y sentido crítico con una argumentación válida y lógica (Maia, 2008).

La Maestría Profesional en Formación Profesional en Red Nacional (ProfEPT) surge como una oportunidad de formación específica en Educación Profesional y RC: 89147

Enlace de acceso: https://www.nucleodoconhecimento.com.br/educacion-es/estudiante-demaestria 
Tecnológica tanto para los empleados de los Institutos Federales como para la población externa, teniendo la producción de conocimiento a través de productos educativos y logros de investigación que se relacionan con el mundo del trabajo. En esta modalidad, el objetivo es la unión de contenidos disciplinarios y conocimientos pedagógicos, la producción académica final debe estar vinculada a situaciones reales de la práctica educativa, y el trabajo final puede ser presentado de diversas maneras (Machado e Urbanetz, 2019; Urbanetz et al., 2020).

Para la producción y publicación del conocimiento científico, la orientación juega un papel fundamental. El asesor que tiene una amplia experiencia en la publicación, se convierte en un mentor para su mentoría, impactando positivamente en la productividad, siendo responsable del progreso del aprendizaje y el éxito en el futuro del estudiante. Es decir, toda la experiencia adquirida durante el proceso de formación tiene un impacto positivo en la vida académica del alumno, las habilidades y destrezas para su publicación se aprenden durante la formación (Falaster et al. 2017).

Según el formulario de evaluación de programas académicos y profesionales (Área 46: Docencia) de la Coordinación para el Perfeccionamiento del Personal de Educación Superior (CAPES):

[...] se evaluará la capacidad mínima de producción pertinente de DP, que podrá involucrar a estudiantes y graduados. Asimismo, se evaluará la adhesión y alineación de la producción indicada por el PPG DP (cuatro producciones) con su área de concentración y líneas de investigación, reflejando la coherencia de la producción con los objetivos e identidad del Programa. [...] Para ello, el PPG indicará hasta cinco producciones bibliográficas y hasta cinco PTT publicadas en el cua ${ }^{\circ}$ periodo en el que participen estudiantes y egresados. [...] la relevancia de la producción indicada en relación con el impacto y el carácter innovador de la producción intelectual, la cobertura local, regional, nacional o internacional, y la satisfacción de las demandas sociales. (Capes, 2020).

La producción académica, tanto del asesor como del mentor, además de la importancia relacionada con la divulgación científica, tiene peso en la evaluación del programa de posgrado stricto sensu y también en su mantenimiento. 


\section{GOL}

Demostrar cuantitativamente la producción académica y educativa de un estudiante de maestría en Educación Profesional y Tecnológica (EPT) del Instituto de Educación, Ciencia y Tecnología (FIPA) de Macapá, Amapá, Amazônia, Brasil.

\section{METODOLOGÍA}

Este artículo expone la producción intelectual desarrollada durante el ProfEPT FIPA.Estas producciones que pueden clasificarse como producción bibliográfica (artículo en periódico, libro) y producción técnica (aplicación - APP, sitio web)

Las encuestas cuantitativas para obtener datos sobre la aplicación y el sitio web utilizaron el período comprendido entre el 12 de abril de 2021 y el 14 de junio de 2021. Los datos sobre la aplicación fueron tomados de la Play Console (Playconsole, 2021) y el sitio web de Google Analytics (Googleanalytics, 2021).

Para los libros y artículos científicos, se utilizó la fecha de publicación original hasta el 14 de junio de 2021. Los datos sobre ellos fueron tomados de los datos en línea (libros y artículos publicados na Revista Multidisciplinar Núcleo do Conhecimento) (Rmnc, 2021) y también por consulta directa de la revista (artículo publicado en Research, Society and Development journal) (Rsd, 2021).

\section{RESULTADOS}

\section{APLICACIÓN EFE (EDUFISEPT)}

La figura 1 muestra el porcentaje de instalaciones de aplicaciones EFE por fuente de tráfico de información entre el 12 de abril y el 14 de junio de 2021. Los datos muestran que el 21,67\% (13 usuarios) llegó a la aplicación a través de la búsqueda dentro de la tienda Google Play y el 78,30\% (47 usuarios) a través de un enlace de terceros. 
Figura 1 - Muestra el porcentaje de instalaciones de aplicaciones EFE por fuente de tráfico de información entre el 12 de abril y el 14 de junio de 2021.

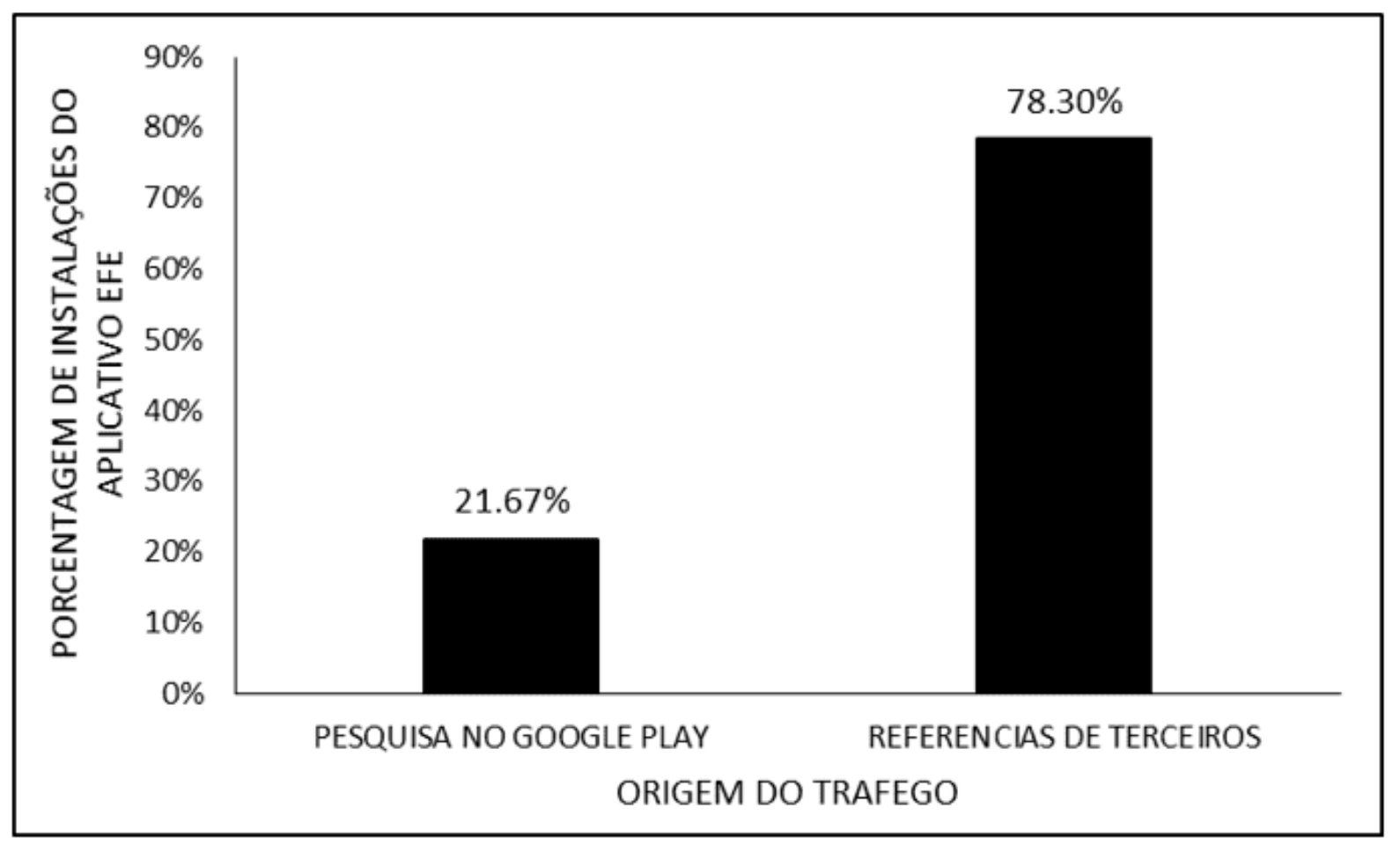

Fuente: Adaptado de Playconsole (2021).

En cuanto al análisis del porcentaje de instalaciones de la aplicación EFE por país del usuario, entre el 12 de abril y el 14 de junio de 2021 se registraron descargas en dos países, con un 98,33\% (59 downloads) de Brasil y un 1,67\% de Portugal (01 downloads) (Figura 2). 
Figura 2 - Muestra el porcentaje de instalaciones de aplicaciones de EFE por país de usuario entre el 12 de abril y el 14 de junio de 2021.

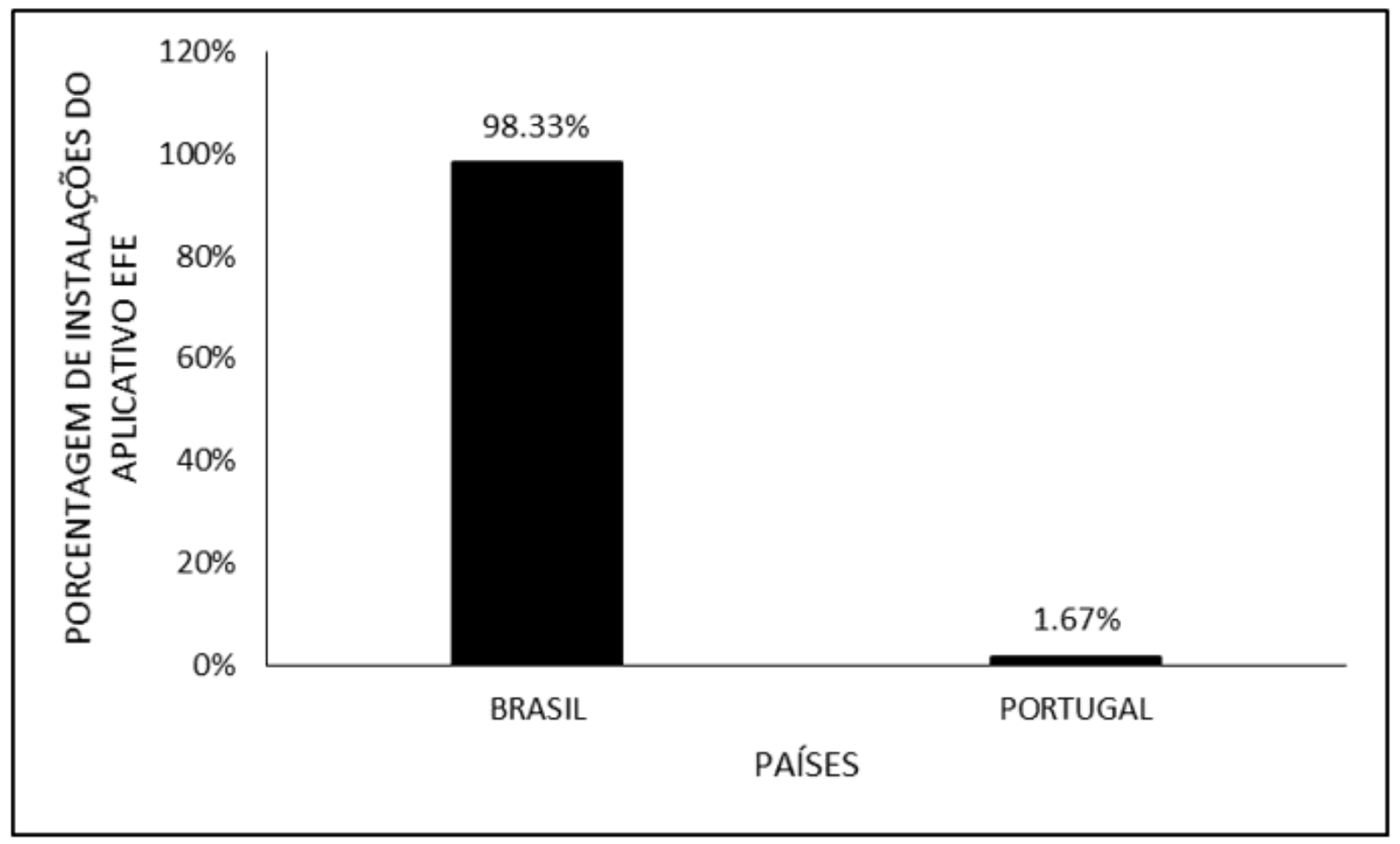

Fuente: Adaptado de Playconsole (2021).

El porcentaje de instalaciones de aplicaciones de efe por visita e instalación de aplicaciones, entre el 12 de abril y el 14 de junio de 2021, se puede ver en la Figura 3.

Del $100 \%$ de los visitantes de la página en la tienda (82 personas), el 73,17\% (60 personas) descargaron la aplicación en su dispositivo móvil (Figura 3). 
Figura 3 - Muestra el porcentaje de instalaciones de aplicaciones de EFE por visita e instalación de aplicaciones entre el 12 de abril y el 14 de junio de 2021.

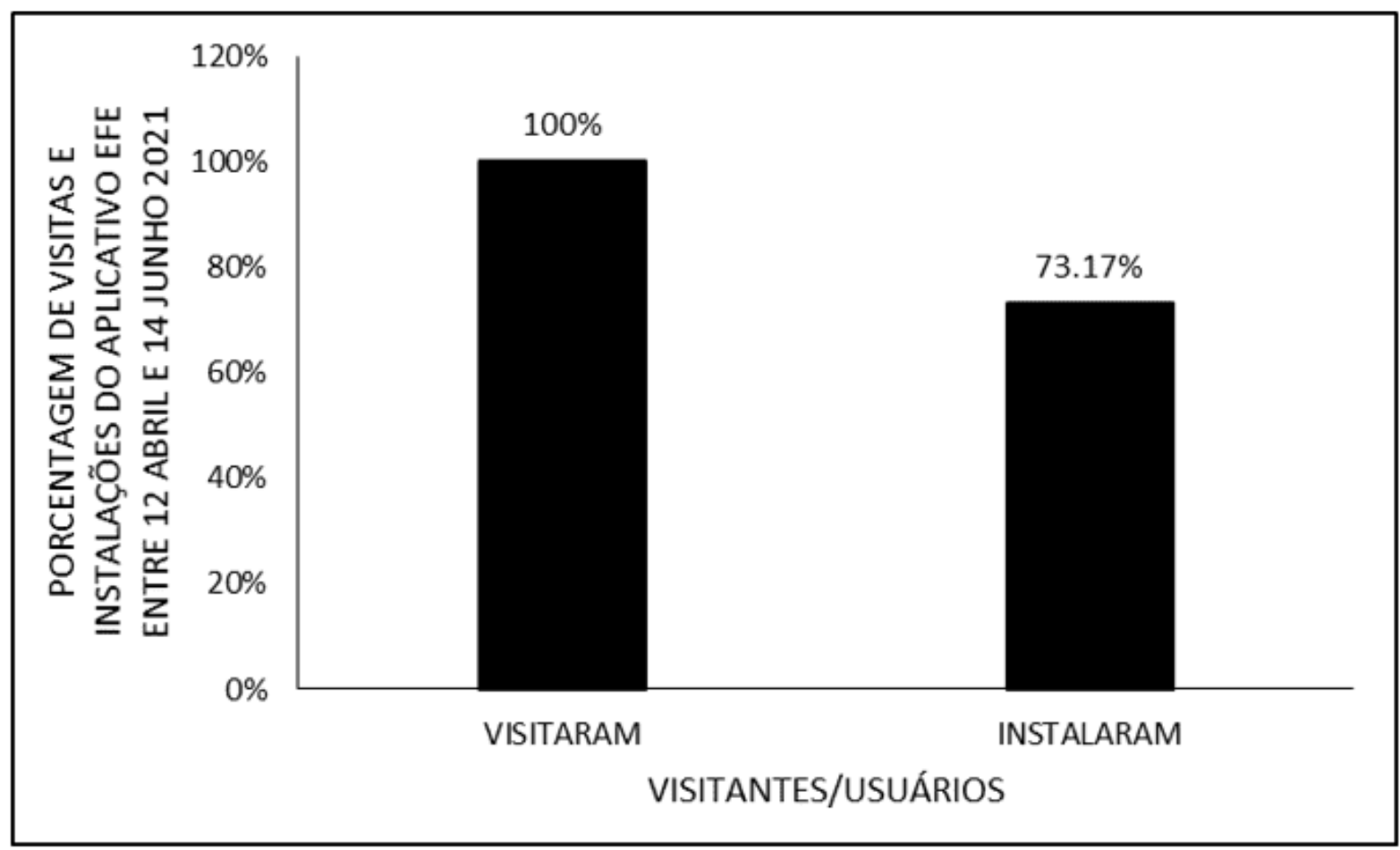

Fuente: Adaptado de Playconsole (2021).

La figura 4 muestra el porcentaje de instalaciones de aplicaciones de EFE por tipos de usuario entre el 12 de abril y el 14 de junio de 2021. Del total (60 usuarios), el $93,33 \%$ (56 personas) son usuarios nuevos y el 6,67\% (04 personas) son usuarios recurrentes dentro de la aplicación, es decir, accedieron a la APP varias veces. 
Figura 4 - Muestra el porcentaje de instalaciones de aplicaciones de EFE por tipos de usuario entre el 12 de abril y el 14 de junio de 2021.

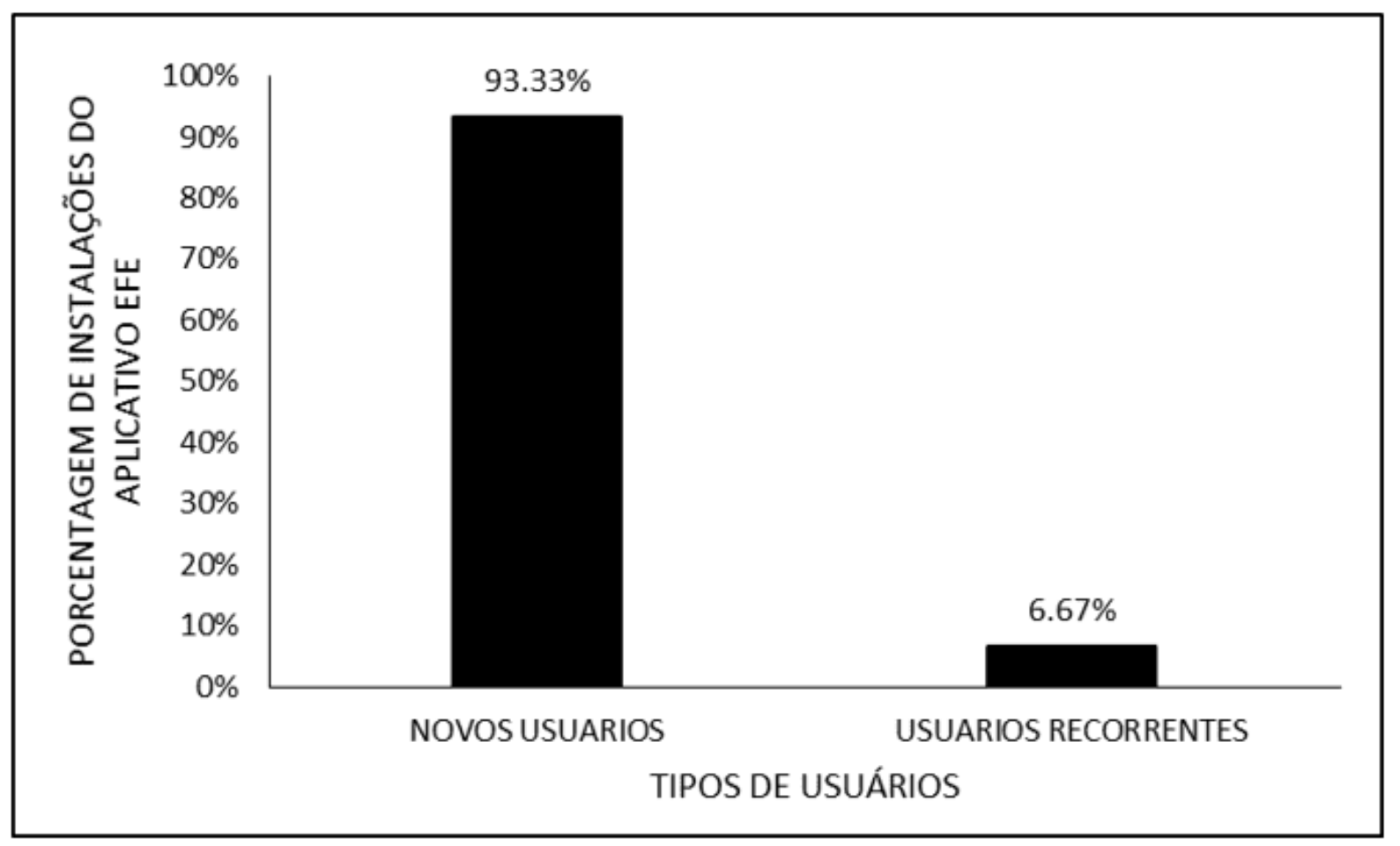

Fuente: Adaptado de Playconsole (2021).

\section{SITIO}

En cuanto al porcentaje de accesos al sitio EduFisEPT por páginas internas del sitio, entre el 12 de abril y el 14 de junio de 2021, la página con más accesos entre los 1399 usuarios (100\%) es prácticas pedagógicas con 30.38\% (425 personas). A continuación la Página de Deportes con 17,23\% (241 personas accediendo), Autores con $13,37 \%$ (187 personas), Colaborar con $13,22 \%$ (185 personas), Juegos y juegos con 7,79\% (109 personas accediendo), Danzas 5,65\% (79 personas), Salud con $5,00 \%$ (70 personas), Ocio con 4,43\% (62 personas) y Peleas con 2,91\% (41 personas) (Figura 5). 
Figura 5 - Muestra el porcentaje de accesos al sitio EduFisEPT por página interna entre el 12 de abril y el 14 de junio de 2021.

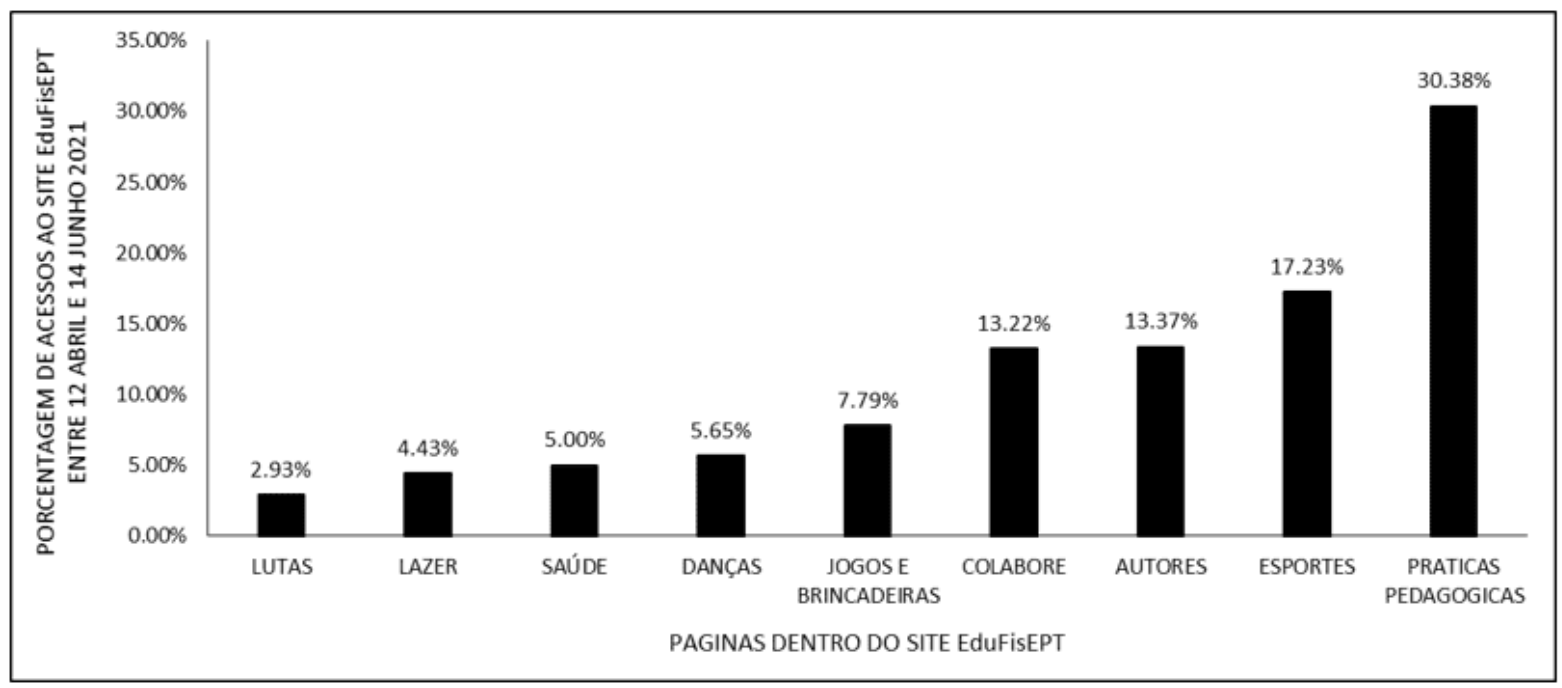

Fuente: Adaptado de GoogleAnalytics (2021).

El porcentaje de accesos al sitio EduFisEPT por países, entre el 12 de abril y el 14 de junio de 2021, sitúa a Brasil con un 93,92\% (1314 personas) de accesos, seguido de Portugal con un 2,64\% (37 personas), Estados Unidos con un 1,50\% (21 personas), Mozambique un 1,07\% (15 personas), un 1,07\%, e Irlanda un 0,86\% (12 personas) (Figura 6). 
Figura 6 - Muestra el porcentaje de accesos al sitio web de EduFisEPT por países entre el 12 de abril y el 14 de junio de 2021.

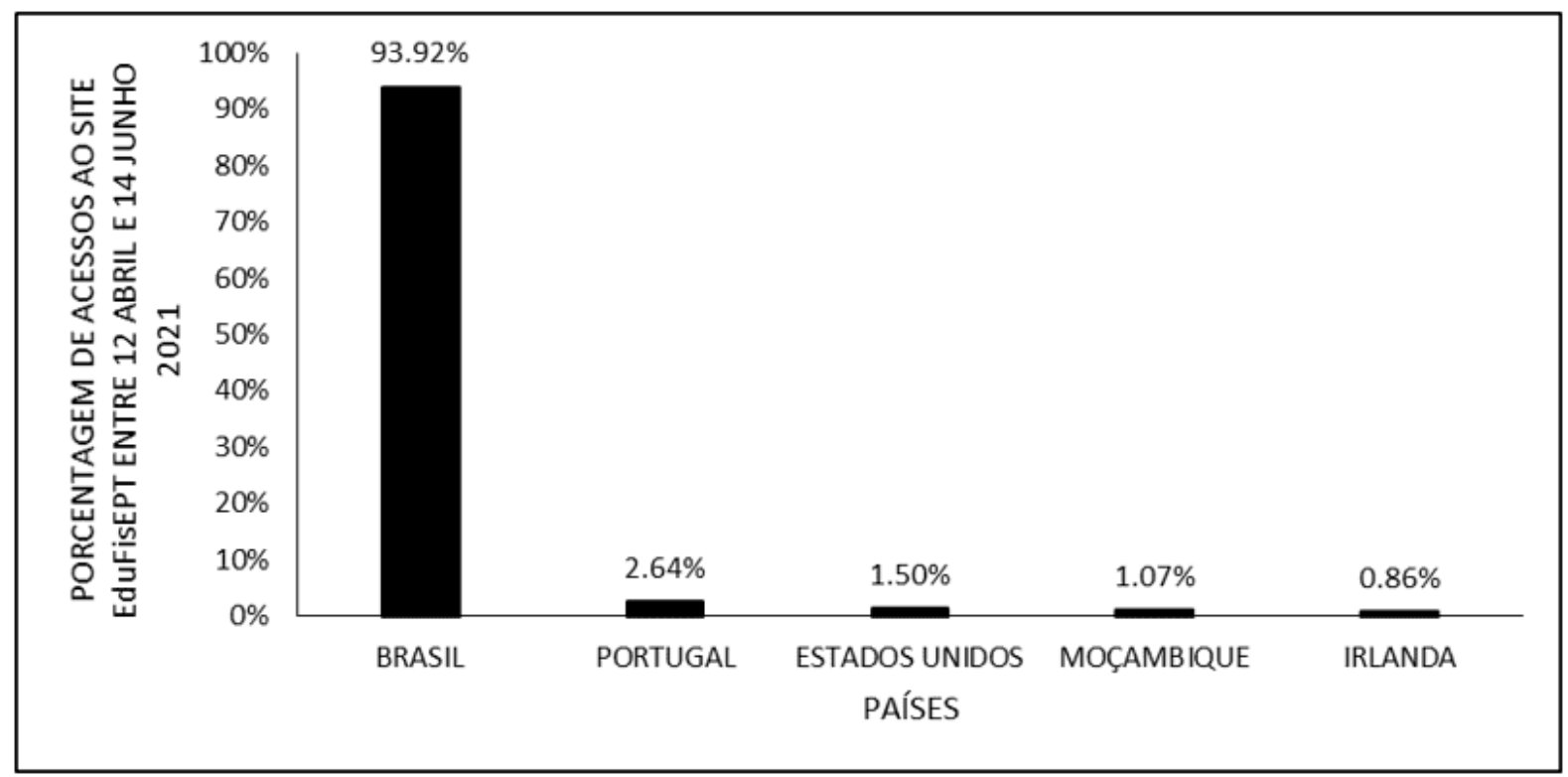

Fuente: Adaptado de GoogleAnalytics (2021).

\section{LIBROS}

La Figura 7 muestra la portada del libro publicado en portugués (BR) el 30 de septiembre de 2020. Desde su publicación, el libro ha acumulado un total de 715 visualizaciones. 
Figura 7 - Muestra la portada del libro publicado en portugués (BR) el 30 de septiembre de 2020.

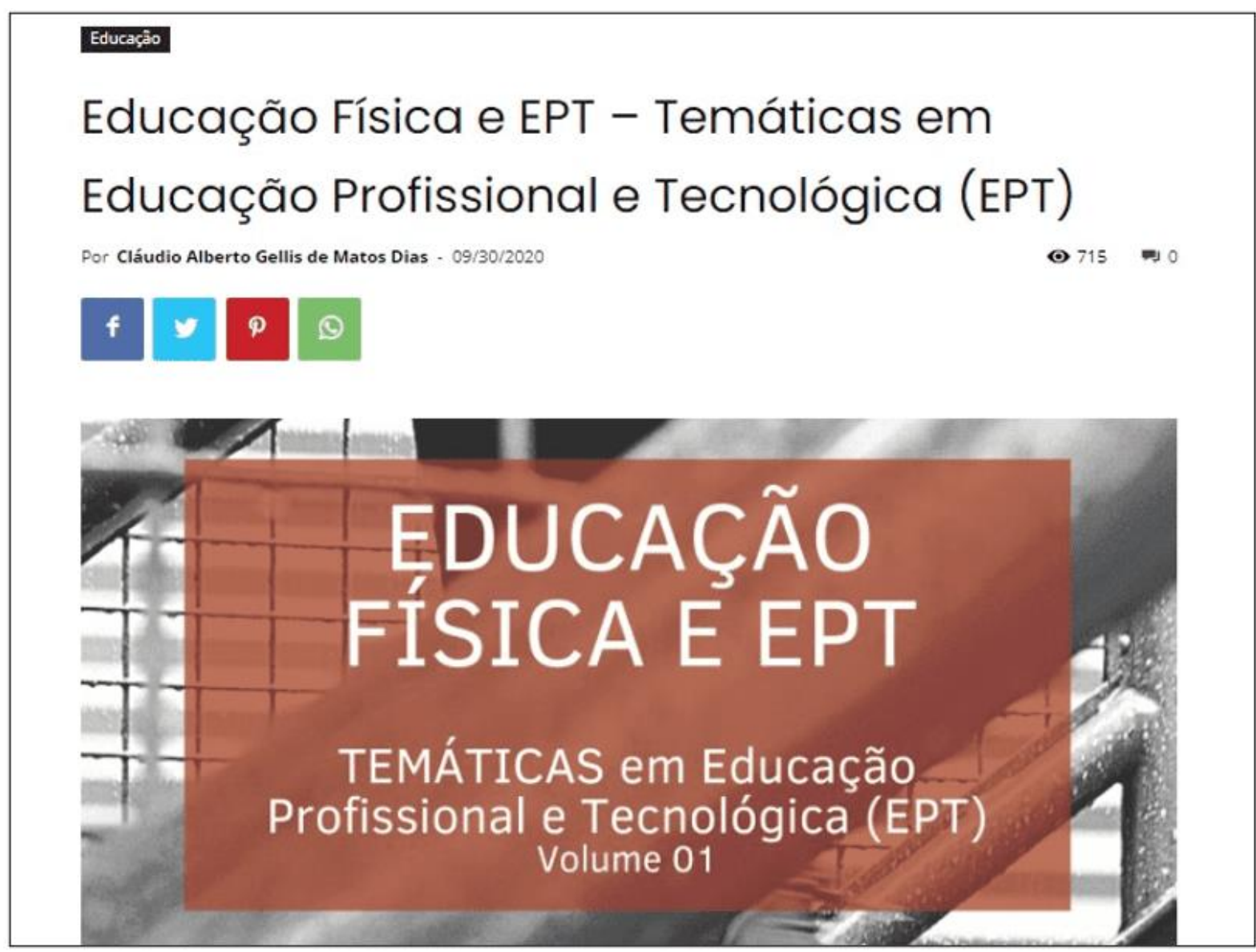

Fuente: Adaptado de Viana y Dias (2020).

El libro publicado en inglés (versión del libro en portugués) el 10 de diciembre de 2020 acumula 248 visualizaciones desde entonces (Figura 8). 
Figura 8 - Muestra la portada del libro publicado en inglés el 10 de diciembre de 2020.

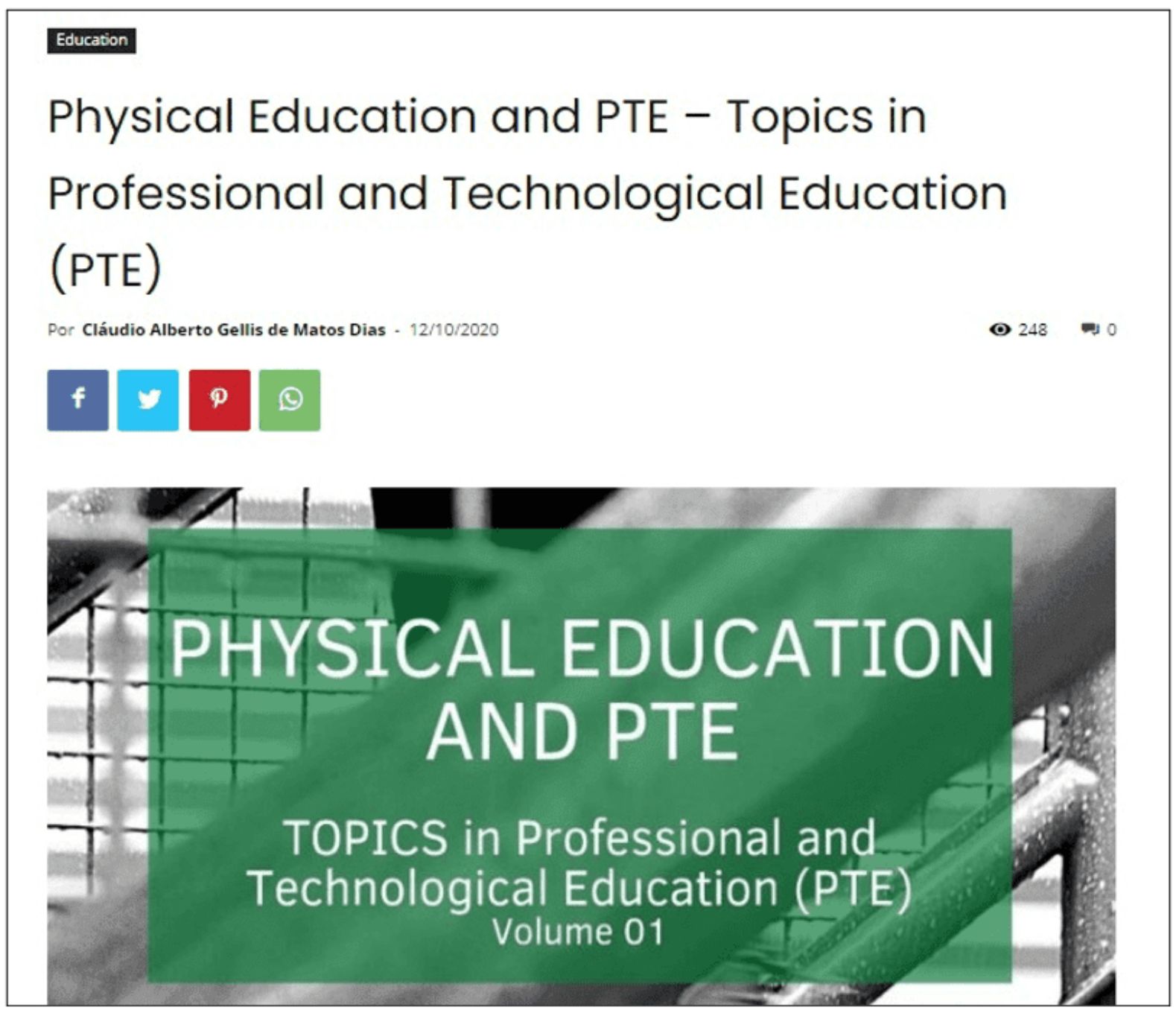

Fuente: Adaptado de Viana y Dias (2020b).

\section{ARTíCULOS PUBLICADOS EN REVISTAS INDEXADAS}

El artículo publicado en la revista Research, Society and Development (Qualis 20132016 B2 y Qualis reference A3) el 2 de julio de 2020 tuvo, según la información de los editores, 91 vistas (Figura 9). 
Figura 9 - Muestra la portada del artículo publicado en la revista Research, Society and Development el 2 de julio de 2020.

\begin{tabular}{|} 
Research, Society and Development, v. 9, n. 8, e239985704, 2020 \\
(CC BY 4.0) | ISSN 2525-3409 | DOI: http://dx.doi.org/10.33448/rsd-v9i8.5704 \\
Quadras de esportes em condições de uso adequado no Brasil: \\
influência no ensino-aprendizagem nas aulas de Educação Física (2015) \\
Sports courts in conditions of proper use in Brazil: influence on teaching-learning in \\
Physical Education classes (2015) \\
Canchas deportivas en condiciones de uso adecuado en Brasil: \\
influencia en la enseñanza-aprendizaje en las clases de Educación Física (2015) \\
Recebido: 16/06/2020 | Revisado: 18/06/2020 | Aceito: 22/06/2020 | Publicado: 02/07/2020 \\
Valderi Nascimento Viana \\
ORCD: https:/orcid.org/0000-0002-0885-5834 \\
Instituto Federal do Amapá, Brasil \\
E-mail: wallderinascimento@gmail.com
\end{tabular}

La Figura 10 muestra la portada del manual sobre la aplicación EFE publicado en la Revista Multidisciplinar Núcleo do Conhecimento (referencia Qualis B3) el 14 de abril de 2021. La publicación en portugués tuvo 244 visitas. 
La Figura 10 - Muestra la portada del manual sobre la aplicación EFE publicado en la Revista Multidisciplinar Núcleo do Conhecimento el 14 de abril de 2021.

\section{Educaçầo Física}

MANUAL DE PRATICAS EM EDUCAÇÃO FÍSICA NA EDUCAÇÃO PROFISSIONAL E TECNOLÓGICA UTILIZANDO O APLICATIVO GRATUITO EFE - EPTFIS

Por Carla Dendasck - RC: 82194 - 14/04/2021

DOI: $10.32749 /$ nucleodoconhecimento.com.br/educacao-fisica/manual-de-praticas

\section{ARTIGO ORIGINAL}

VIANA, Valderi Nascimento [1], FECURY, Amanda Alves [2], OLIVEIRA, Euzébio de [3], DENDASCK, Carla Viana ${ }^{[4]}$, DIAS, Claudio Alberto Gellis de Mattos ${ }^{[5]}$

Fuente: Adaptado de Viana et al. (2021).

El manual se publicó en seis idiomas más, además del portugués. La Tabla 1 muestra el número de accesos (y la URL) al manual sobre la aplicación EFE publicado en la Revista Multidisciplinar Núcleo do Conhecimento hasta el 14 de junio de 2021, por idioma. En total, desde la publicación hasta el 14 de junio de 2021 ha habido 374 visualizaciones. 
Tabla 1 - Muestra el número de accesos (y url) al manual sobre la aplicación EFE publicado en la Revista Multidisciplinar Núcleo do Conhecimento hasta el 14 de junio de 2021, por idioma.

\begin{tabular}{|c|c|c|}
\hline IDIOMA & $\begin{array}{c}\text { NÚMERO } \\
\text { DE } \\
\text { ACESSOS }\end{array}$ & LINK DE ACESSO \\
\hline PORTUGUES & 244 & $\begin{array}{l}\text { https://www.nucleodoconhecimento.com.br/e } \\
\text { ducacao-fisica/manual-de-praticas }\end{array}$ \\
\hline INGLES & 20 & $\begin{array}{l}\text { https://www.nucleodoconhecimento.com.br/e } \\
\text { ducation-physics-en/manual-of-practices }\end{array}$ \\
\hline ESPANHOL & 24 & $\begin{array}{l}\text { https://www.nucleodoconhecimento.com.br/e } \\
\text { ducacion-fisica-es/manual-de-practicas }\end{array}$ \\
\hline FRANCÊS & 23 & $\begin{array}{l}\text { https://www.nucleodoconhecimento.com.br/e } \\
\text { ducation-physics-fr/manuel-de-pratiques }\end{array}$ \\
\hline ITALIANO & 26 & $\begin{array}{l}\text { https://www.nucleodoconhecimento.com.br/e } \\
\text { ducazione-fisica-it/manuale-di-pratiche }\end{array}$ \\
\hline ALEMÃO & 21 & $\begin{array}{l}\text { https://www.nucleodocon hecimento.com.br/b } \\
\text { ildung-physik-de/handbuch-der-praktiken }\end{array}$ \\
\hline RUSSO & 16 & $\begin{array}{l}\text { https://www.nucleodocon hecimento.com.br/o } \\
\text { бразование-физика-ru/пособие-по-практике, }\end{array}$ \\
\hline TOTAL & 374 & \\
\hline
\end{tabular}

Fuente: Adaptado de Viana et al. (2021), Viana et al. (2021b), Viana et al. (2021c), Viana et al. (2021d), Viana et al. (2021e), Viana et al. (2021f), Viana et al. (2021g).

\section{DISCUSIÓN}

Dentro del ámbito científico, toda teoría y metodología busca acercar al alumno al conocimiento producido por la ciencia. Dentro del proceso de capacitación, el contacto con la redacción de artículos, la construcción de sitios web y la aplicación, la construcción de investigaciones, el análisis y la difusión de datos, demostraron ser de gran importancia. En cada producción se está investigando una realidad, que establece una conexión con situaciones y contextos socioculturales. La RC: 89147

Enlace de acceso: https://www.nucleodoconhecimento.com.br/educacion-es/estudiante-demaestria 
contextualización del conocimiento es una herramienta para el aprendizaje significativo. Es oportunista, nueva experiencia o resignificación del conocimiento, es desarrollar la capacidad de analizar, hipótesis, sentido crítico dentro de una estructura actual, ya que se produce la interacción con hechos y fenómenos de la vida cotidiana (Yamaguchi e Furtado, 2019).

Todos los materiales publicados, física o on-line, sirvencomo medio de divulgación científica. En el caso de la aplicación todos los contenidos cubiertos se basan en el conocimiento científico, mediante un acceso fácil y sencillo. El profesor adquiere nuevos conocimientos y puede ponerlos en práctica dentro de la escuela en tiempo real. Según Rossi (2019)

Queda acercar la ciencia de los artículos y congresos a la vida de los brasileños que tanto necesitan casi todo con respecto a la salud, la calidad de vida, la sostenibilidad, la movilidad, los usos de la tecnología y la ciudadanía.

Así, tanto a nivel teórico (artículos, libros, página web) como la aplicación de la app sirven de estímulo a la experiencia científica, es pensar en la ciencia articulada con la realidad, el acceso y el lenguaje accesible a todo aquel que busque conocimiento. La difusión va más allá de tomar un conocimiento sistematizado, también sirve para deleitar, motivar y estimular al otro. (Rossi, 2019).

En educación física, la visión de una práctica refinada en técnica por técnica o rendimiento deportivo sigue siendo muy fuerte. El rendimiento y la técnica deben ser considerados por el profesor, pero no vistos como prioridades y deben estar dentro de un estándar de excelencia sin respetar la individualidad del estudiante. Dentro de una perspectiva de formación global, las clases de educación física deben trabajar su objeto de estudio en una dimensión amplia, ya que los estudiantes se insertan dentro de una sociedad que tiene una cultura y que produce significados, así como revela contradicciones de comportamientos, actitudes que deben ser abordados / considerados (Coutinho, 2010; Viana et al., 2020). 
Así, con las producciones científicas basadas en las ciencias humanas y sociales que solo contribuyen al área de la educación física, superando solo el uso de las ciencias de la salud, existe la posibilidad de situar la educación física en el mismo nivel de relevancia e importancia de otros componentes del currículo escolar (Balbino e Urt, 2018). Durante el proceso de búsqueda, los artículos y libros fueron traducidos a otros idiomas. Esta actitud es importante para ampliar el alcance de la investigación, y la producción puede estar relacionada con la literatura nacional e internacional, mostrando una capacidad de comunicación, acceso, cooperación y apoyo con lo que está siendo producido por el mundo dentro de la educación física (Coutinho, 2010).

En cuanto a la producción científica en el Norte, Pereira e Silveira (2019) realizaron un análisis de la producción científica de investigadores de la Región Norte en el Encuentro Nacional de Investigación en Educación Científica e informaron que el Norte tiene la menor cantidad de investigación presente en el evento. El estudio también muestra que Amapá no realizó ninguna presentación y trabajo en las ediciones de 2009, 2011, 2013, 2015 y 2017. Según el estudio, el mayor o menor número de producciones está relacionado con la localidad del campus universitario, ya que son responsables de la mayoría de las actividades científicas. Corroborando el estudio antes mencionado, Sidone et al. (2016) al hacer un análisis de la producción de artículos entre los años 2007-2009 de las regiones Sur y Sureste indica que estos fueron responsables de más de tres cuartas partes de la producción total. Otras regiones como el noreste $15 \%$ y el medio oeste y el norte juntos no alcanzaron el $10 \%$ del total nacional. Además de la concentración de universidades en las regiones Sur y Sureste, otro factor que apunta a esta producción es la mayor disponibilidad de recursos humanos.

\section{CONCLUSIONES}

Durante el máster, la producción científica en diversas formas resultó necesaria durante el proceso de formación. Uniendo la teoría con la práctica de la escritura, facilitó la producción de la disertación que debía presentarse al final del curso. A través 
del proceso de organización, la planificación de las ideas dio lugar a un nuevo pensamiento estructurado de acuerdo con los estándares de la academia, pero que no se limita a este espacio y las normas.

Teniendo un aporte que va más allá de la escritura científica, durante este camino de aprendizaje fue ampliamente, es decir, creación de aplicaciones, sitios web, libros, artículos, metodologías de prácticas pedagógicas. Toda esta experiencia impactará positivamente en el futuro como investigador y profesional de la educación física.

En este contexto, toda la producción durante la maestría también contribuye cuantitativa y cualitativamente a la producción científica de la región norte, impactando positivamente en ella y en el programa de maestría. Además de la importancia en la contribución de la creación y divulgación científica, las producciones tienden a impactar positivamente en la evaluación y mantenimiento del programa PROFEPTFIPA, realizado por la CAPES.

\section{REFERENCIAS}

BALBINO, S. I.; URT, S. D. C. Prática Pedagógica em Educação Física para a Educação Integral em Tempo Integral. Pensar a Prática, v. 21, n. 4, p. 775-785, 2018.

CAPES. Ficha de Avaliação - Programas Acadêmicos e Profissionais Área 46: Ensino. Brasília DF, 2020. Disponível em: < https://www.gov.br/capes/pt-br/centraisde-conteudo/FICHA_ENSINO.pdf >. Acesso em: 14 Jun 2021.

COUTINHO, R. X. A influência da Produção Científica nas Práticas de Professores de Educação Física, Ciências e Matemática em Escolas Públicas Municiais de Uruguaiana - RS. 2010. 94p. (Mestrado). Universidade Federal de Santa Maria, Santa Maria RS.

FALASTER, C.; FERREIRA, M. P.; GOUVEA, D. M. R. D. O efeito da publicação científica do orientador na publicação dos seus orientados. Revista de Administração Contemporânea, v. 21, n. 4, p. 458-480, 2017. 
GOOGLEANALYTICS. Google Analytics Aplaticativo Oficial EFE - Pagina Inicial. 2021. Disponível em: < https://analytics.google.com/analytics/web/?authuser=1\#/p265457828/reports/default home >. Acesso em: 14 Jun 2021.

MACHADO, M. F. R. C.; URBANETZ, S. T. Mestrado profissional em educação profissional e tecnológica no Instituto Federal do Paraná: desafios e expectativas. Atos de Pesquisa em Educação, v. 14, n. 2, p. 885-903, 2019.

MAIA, R. T. A importância da disciplina de metodologia científica no desenvolvimento de produções acadêmicas de qualidade no nível superior. Revista Urutágua, v. 14, n. 1, 2008.

PEREIRA, T. Z. M.; SILVEIRA, C. A Produção Acadêmica Da Região Norte: Uma Análise Na Ata Do Xi Encontro Nacional De Pesquisa Em Educação Em Ciências. REAMEC - Rede Amazônica de Educação em Ciências e Matemática, v. 7, n. 2, p. 245-260, 2019.

PLAYCONSOLE. Google Play Console Painel - EFE EduFIsEPT. 2021. Disponível em:

https://play.google.com/console/u/0/developers/4760644030222400117/app/497210 7118591571772/app-dashboard?timespan=thirtyDays\&showKpiMenu=null >. Acesso em: 14 Jun 2021.

RMNC. Revista Multidisciplinar Nucleo do Conhecimento. São Paulo SP, 2021. Disponível em: < https://www.nucleodoconhecimento.com.br/ >. Acesso em: 14 Jun 2021.

ROSSI, A. V. Sobre A Valorização Das Contribuições Da Divulgação Científica No Brasil. In: VOIGT, C. L. (Ed.). O ensino de química. Ponta Grossa PR: Atena Editora, v.1, 2019. cap. 11, p.136-148. 
RSD. Research, Society and Development. Vargem Grande paulista SP, 2021. Disponível em: < https://rsdjournal.org/index.php/rsd >. Acesso em: 14 Jun 2021.

SIDONE, O. J. G.; HADDAD, E. A.; MENA-CHALCO, J. P. A ciência nas regiões brasileiras: evolução da produção e das redes de colaboração científica. Transinformação, v. 28, n. 1, p. 15-32, 2016.

URBANETZ, S. T.; CASSIANO, E. L.; BETTONI, V. O Mestrado Profissional Em Educação Profissional E Tecnológica-Profept-E O Significado Dessa Oferta De Formação Em Pós Graduação No Brasil. Movimento-revista de educação, v. 7, n. 14, 2020.

VIANA, V. N.; DIAS, C. A. G. D. M. Educação Física e EPT - Temáticas em Educação Profissional e Tecnológica (EPT). Macapá AP: 2020. 45 p. ISBN 97865-86069-90-7. Disponível em:

https://www.nucleodoconhecimento.com.br/livros/wp-content/uploads/2020/09/livroeducacao-Fisica-e-EPT-educacao-profissional-e-tecnologica-2020.pdf >.

. Physical Education and PTE - Topics in Professional and Technological Education (PTE). Macapá AP: 2020b. 45 p. ISBN 978-65-86069-89-1. Disponível em: < https://www.nucleodoconhecimento.com.br/livros/en/education/physicaleducation-and-pte $>$.

VIANA, V. N. et al. Quadras de esportes em condições de uso adequado no Brasil: influência no ensino-aprendizagem nas aulas de Educação Física (2015). Research, Society and Development, v. 9, n. 8, p. e239985704, 2020. Disponível em: < https://rsdjournal.org/index.php/rsd/article/view/5704/4727 >.

VIANA, V. N. et al. Manuel de pratiques en éducation physique en éducation professionnelle et technologique utilisant l'application gratuite EFE - EPTFIS. Revista Científica Multidisciplinar Núcleo do Conhecimento, v. 5, p. 4, 2021d. Disponível 
em: < https://www.nucleodoconhecimento.com.br/education-physics-fr/manuel-depratiques $>$.

. Manuale di pratiche in educazione fisica nell'educazione professionale e tecnologica utilizzando l'applicazione gratuita EFE - EPTFIS. Revista Científica Multidisciplinar Núcleo do Conhecimento, v. 5, n. 4, p. 65-76, 2021e. Disponível em: < https://www.nucleodoconhecimento.com.br/educazione-fisica-it/manuale-dipratiche $>$.

- Handbuch der Praktiken im Sportunterricht im beruflichen und technologischen Unterricht mit der kostenlosen Anwendung EFE - EPTFIS. Revista Científica Multidisciplinar Núcleo do Conhecimento, v. 5, n. 4, p. 6576, 2021f. Disponível em: < https://www.nucleodoconhecimento.com.br/bildungphysik-de/handbuch-der-praktiken $>$.

. Пособие по практике физического воспитания в профессиональном и технологическом образовании с использованием бесплатного приложения EFE EPTFIS. Revista Científica Multidisciplinar Núcleo do Conhecimento, v. 5, n. 4, p. 65-76, 2021g.

Disponível em:

https://www.nucleodoconhecimento.com.br/образование-физика-ru/пособие-попрактике >.

VIANA, V. N. et al. Manual De Praticas Em Educação Física Na Educação Profissional E Tecnológica Utilizando O Aplicativo Gratuito EFE - EPTFIS. Revista Científica Multidisciplinar Núcleo do Conhecimento, v. 5, n. 4, p. 65-76, 2021. Disponível em: $\quad<$ https://www.nucleodoconhecimento.com.br/educacao-fisica/manual-depraticas $>$.

. Manual Of Practices In Physical Education In Professional And Technological Education Using The Free Application EFE - EPTFIS. Revista Científica Multidisciplinar Núcleo do Conhecimento, v. 5, n. 4, p. 65-76, 2021b. Disponível 
em: < https://www.nucleodoconhecimento.com.br/education-physics-en/manual-ofpractices $>$.

. Manual De Prácticas En Educación Física En Educación Profesional Y Tecnológica Utilizando La Aplicación Gratuita EFE - EPTFIS. Revista Científica Multidisciplinar Núcleo do Conhecimento, v. 5, n. 4, p. 65-76, 2021c. Disponível em: < https://www.nucleodoconhecimento.com.br/educacion-fisica-es/manual-depracticas $>$.

YAMAGUCHI, K. K. D. L.; FURTADO, M. A. S. A Escrita Científica Como Aprendizagem Contextualizada: Uma Abordagem Metodológica A Partir De Um Experimento De Mistura De Cores In: VOIGT, C. L. (Ed.). O ensino de química. Ponta Grossa PR: Atena Editora, v.1, 2019. cap. 12, p.149-158.

Enviado: Junio de 2021.

Aprobado: Junio de 2021. 